\title{
The Phantom versus The Falcon: Force Feedback Magnitude Effects on User's Performance during Target Acquisition
}

\author{
Lode Vanacken, Joan De Boeck, and Karin Coninx \\ Hasselt University - tUL - IBBT , Expertise Centre for Digital Media (EDM) \\ Wetenschapspark 2, B-3590 Diepenbeek, Belgium \\ \{lode.vanacken, joan. deboeck, karin. coninx\}@uhasselt. be
}

\begin{abstract}
Applying force feedback applications in a therapy environment allows the patient to practice in a more independent manner, with less intervention of the therapist. Currently however, high-end devices such as the Phantom or the HapticMaster are far too expensive to provide a device per patient. Recently Novint launched a low-cost haptic device for the gaming market: the Falcon. In this paper we report on an experiment that we conducted in order to compare the Falcon and the Phantom, based on a Fitts' law targeting task. We deduced physical parameters such as inertia and damping, which were found to be different for the devices. Although from a velocity analysis these differences can be clearly seen, it turns out that the influence of different forces does not show significant differences when taking completion time and error rate into account. From a subjective experiment, we can learn that users allow the Falcon to produce slightly higher forces than the Phantom before forces are judged as too strong.
\end{abstract}

\section{Introduction}

Haptic interfaces are applied in an increasing number of domains, going from training to entertainment. In a recent pilot study, we used a Phantom Premium 1.0 haptic device (sold by Sensable Technologies) as a force feedback input device in a rehabilitation program for the upper limbs of Multiple Sclerosis (MS) patients [2] (figure 1). Three simple game-like applications were developed, which were included in the patient's therapy during a period of four weeks. The generated force feedback was meant to support, assist or resist patients according to their individual capabilities. Based on the pilot study, this research is continued in a research project with a workplan for basic and applied research in a multidisciplinary consortium.

The benefit of bringing force feedback to a therapy environment is that training sessions can be finely tailored to the patient's need, with a minimal intervention of the therapist. The ultimate goal in this approach is a setup that can be placed at the patient's home, where the follow-up is mainly done remotely [4]. Although the Phantom haptic device we used in the pilot study contributed to 


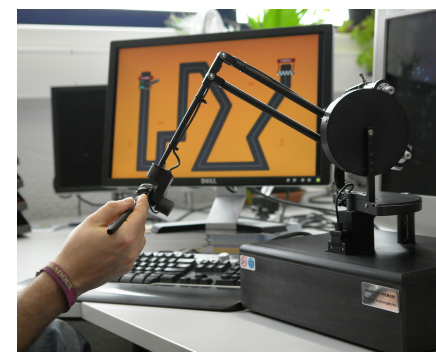

(a)
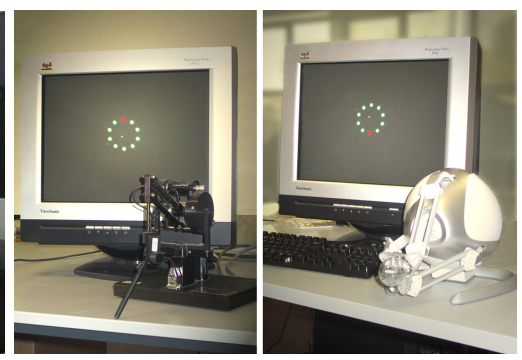

(b)

Fig. 1. (a) One of the games of the rehabilitation project: patients have to keep the car in the middle of the road while the force feedback can be used guide them. (b) Setup of the experiments: Phantom and Falcon with the ISO 9241-9 tapping circle.

promising results, it is very expensive. Providing an individual MS patient with his/her Phantom at home is very unlikely for the next few years.

Recently a new haptic device, the Falcon (by Novint inc), has been launched. The device focusses on a broad consumer market especially for gaming purposes, but has also been sparsely used in some rehabilitation projects [1]. Although it can be expected that this device will have less optimal device characteristics compared to the more expensive Phantom, its current pricing is by far better keeping a home setup in mind. Moreover, a comparison of the devices can also contain interesting information for other application types or interaction techniques.

Due to the different characteristics, a force may be felt differently on different devices, at its turn having implications on the performance and/or the satisfaction of the user or patient. Few studies currently exist that compared haptic devices for certain tasks $[9,6]$. Usually, focus is only on the performance of the user with regard to task completion time, but it is also important to take perception into account as well. Both Yu et al. [9] and Harders et al. [6] found small but non-significant differences with regard to completion time and subjective preference. These experiments only compared the devices using a user's task, whereas we also perform an analysis of their mechanical parameters.

In order to investigate whether or not we have to take the Falcon into account as a possible alternative in our rehabilitation setup, we report on an experiment in which we compare the devices (Phantom Premium 1.0 and a Novint Falcon). In section 2 we deduce the main parameters, mass and damping for the devices. Afterwards section 3 and 4 compare the devices in two target acquisition experiments, respectively targeted towards objective and subjective measures.

\section{Phantom versus Falcon}

The Phantom Premium 1.0 haptic device is a high-end device with prices starting from 24,250 dollar (price from early 2010). On the other hand, the Falcon haptic 

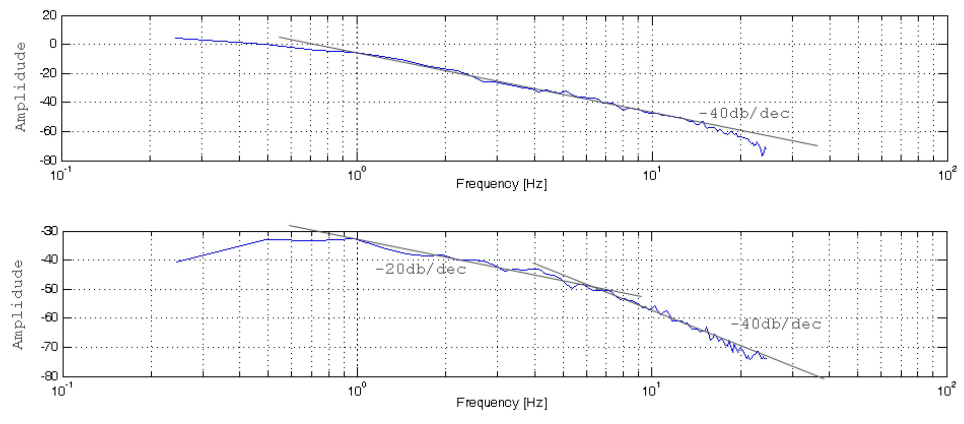

Fig. 2. Transfer functions according to the $\mathrm{X}$-axis for the Phantom premium 1.0 (upper plot) and the Falcon (lower plot).

device is a very low cost haptic device (180 dollar, early 2010) focusing on the consumer market. Both devices are impedance controlled devices, measuring the user's movement at the input and producing a force at the output.

The Phantom premium 1.0 has a workspace of about $25 \times 18 \times 12 \mathrm{~cm}(W \times$ $H \times D)$. The Falcon's workspace is somewhat smaller with $10 \times 10 \times 10 \mathrm{~cm}$. They produce comparable force magnitudes. Besides these general specifications, found on the data sheets, we would like to know parameters such as the damping (mainly caused by friction) and inertia (apparent mass at the device's handle), because the result of the applied forces can be different when a device has a higher inertia or a higher friction.

To achieve this, we a parametric identification using the transfer function had been applied as described by Pintelon et al. [8]. For the devices, white noise in the form of random forces was applied at the input (motors). While the device was in open loop (not held by a user) we measured the position of the end-effector (output).

The transfer functions (ouput/input) in the frequency domain for the devices are given in Figure 2. The phase diagrams have been omitted as we don't use them during the identification process.

Given that the general transfer function is given by $\frac{1}{m s^{2}+b s}$ we know

$$
Y_{(d b)}=\left|20 \log \left(\frac{1}{m s^{2}+b s}\right)\right|
$$

where $m$ can be identified as the mass, $b$ as the damping, and $s$ as the Laplace transform parameter. Two poles can be found, resulting in a transfer function that has a slope of $-20 \mathrm{db} / \mathrm{dec}$ starting from the pole at $0 \mathrm{~Hz}$, and a slope of $-40 \mathrm{db} / \mathrm{dec}$ after the second pole (see Figure 2).

Elaborating the formula towards $f(s=j \omega=2 \pi j f)$, and solving for $m$ and $b$ gives us:

$$
m=\left|\frac{-1}{(2 \pi f)^{2} \cdot 10^{\frac{-Y_{d b}}{20}}}\right| \quad \text { and } \quad b=\left|\frac{-j}{(2 \pi f) \cdot 10^{\frac{-Y_{d b}}{20}}}-(2 \pi j \cdot m \cdot f)\right|
$$


For calculating $m, f$ is a frequency high enough to ignore the influence of the damping and $Y$ is the amplitude read on the graph (in db). For calculating $\mathrm{b}, f$ is a frequency, small enough to minimise the influence of $m$ and $Y$ is again the amplitude read on the graph in db.

The results of the identification can be found in figure 3(a). The values indicated with an asterisk $\left(^{*}\right)$ could not be identified with sufficient accuracy. For the devices, the transfer functions for the y-axis were too noisy to make a decent estimation. For the Phantom, it appears that the damping (mainly caused by the friction) is small compared to the influence of the inertia. Therefore, it is very difficult to make good estimations for this parameter. It may attract the attention that the mass of the phantom for the z-axis is significantly higher, but this may not be surprising because movements around y and $\mathrm{z}$ cause the motors to move. With the Falcon, the friction appears to be the main factor. As a result, the mass can only be identified using a higher frequency (in order to be able to ignore the friction), resulting in a less accurate result.

The most important conclusion for the purpose of this paper, is the fact that the Falcon appears to have a higher damping. We may expect that this will have an influence on the device's behaviour. As the inertia differs not so much (at least not for the z-axis), its influence will be less pronounced.

\section{Objective Comparison}

One of the main purposes of our rehabilitation program is increasing the patient's 'motor control'. Consequently, 'target acquisition' is an important aspect of this training. Fitts' law tasks therefore appear to be suitable for our comparison experiment. Recently, we performed a Fitts' law test (in a different context) in order to investigate how different forces with different duration, amplitude and shape could influence the user's performance [3]. We believe the different force shapes and durations in this former experiment are suitable to compare haptic devices.

From the previous experiment, we could learn that the performance deterioration was a result of a stabilising action of the user, reducing the oscillation caused by the force bump by 'suspending' the targeting movement. As mass and damping have a direct influence on the oscillation, it may sound obvious that other device parameters, may lead to different behaviour. Hence, a similar experiment has been set up comparing the Phantom and the Falcon devices.

\subsection{Apparatus, Procedure and Participants}

Apparatus A Phantom premium 1.0 with stylus and gimbal encoders and a Falcon haptic display was used. The visual output was provided by a 19-inch monitor. For the devices, a control display unitary gain was used.For validition purposes, before starting the experiment, extra care had been taken in calibrating the input devices. Using a 'forcemeter', we measured the influence of the gravity on the grips of the devices (for gravity compensation), as well as the gain factor 


\begin{tabular}{|c|c|c|}
\hline & Phantom & Falcon \\
\hline$m_{x}$ & 0.06 & 0.17 \\
$b_{x}$ & 0.51 & 8.20 \\
\hline$m_{y}$ & $*$ & $*$ \\
$b_{y}$ & $*$ & $*$ \\
\hline$m_{z}$ & 0.41 & 0.23 \\
$b_{z}$ & $*$ & 9,28 \\
\hline
\end{tabular}

(a)

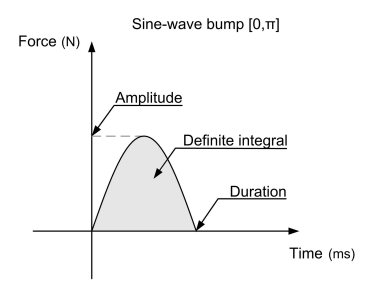

(b)

Fig. 3. (a) The identified parameters $(\operatorname{mass}(m)$ and damping $(b))$ of the Phantom and the Falcon. (b) Illustration of the force evolution of a sinusoidal haptic bump over time.

between the force requested in the software (CHAI3D API) and the final result at the device. The forces for the Phantom were quite correct, for the Falcon a multiplication factor of 3 had to be used, to achieve the same output.

Procedure A simple multidirectional point-select task, as described in ISO 92419, was used for this experiment. Ten target circles are placed in a circle on the screen (see Figure 1(b)). The diameter of the circle is determined at $6 \mathrm{~cm}$ and the size of each target is $0.7 \mathrm{~cm}$ (we use physical measures rather than pixels, since pixel sizes vary from display to display). This task has a Fitts' index of difficulty of 3.26 bits. The value is chosen to be comparable to the task difficulty of a typical icon selection task.

During the test, the ten target circles were highlighted one after the other and users were requested to select the highlighted target 'as most efficiently' (rapidly and accurately) as possible, by pointing and clicking. Highlighting is altered between opposite sides of the circle so that it requires the user to make equally distributed movements among all directions with a maximum distance between the targets.

Other measures (2D force feedback plane, visual feedback, ... ) were made to ensure experimental validity, these are elaborately discussed in [3] and omitted here due to space constraints.

Finally, force feedback appearing in the form of a force bump with given shape, duration and amplitude was activated when half-way in the path to the next target. Half-way the path is calculated using a simple Euclidian distance. Note that this activation strategy serves as a distractor without any purpose of being beneficial, but similar forces can be used for instance to indicate a transition between two regions.

Participants Seventeen healthy male and one female unpaid volunteers, ranging in age from 20 to 28 with an average of 22.7 , served as participants in this experiment. They were recruited among computer science students and had not taken part in any of our previous experiments. All participants were righthanded and used their dominant hand during the experiment. We preferred 


\begin{tabular}{|c|c|c|c|c|}
\hline $\begin{array}{c}\text { Force } \\
\text { Integral }\end{array}$ & $\begin{array}{l}\sin _{[0, \pi]} \\
(75 \mathrm{~ms})\end{array}$ & $\begin{array}{c}\text { sqr } \\
(40 \mathrm{~ms})\end{array}$ & $\begin{array}{c}\sin _{[0, \pi]} \\
(110 \mathrm{~ms})\end{array}$ & $\begin{array}{r}\sin _{[0,2 \pi]} \\
(75 \mathrm{~ms}) \\
\end{array}$ \\
\hline 0.0 & $0.0 \mathrm{~N}$ & $0.0 \mathrm{~N}$ & $0.0 \mathrm{~N}$ & $0.0 \mathrm{~N}$ \\
\hline 9.55 & $0.2 \mathrm{~N}$ & $0.24 \mathrm{~N}$ & $0.14 \mathrm{~N}$ & $0.2 \mathrm{~N}$ \\
\hline 19.10 & $0.4 \mathrm{~N}$ & $0.48 \mathrm{~N}$ & $0.27 \mathrm{~N}$ & $0.4 \mathrm{~N}$ \\
\hline 28.65 & $0.6 \mathrm{~N}$ & $0.72 \mathrm{~N}$ & $0.41 \mathrm{~N}$ & $0.6 \mathrm{~N}$ \\
\hline 38.20 & $0.8 \mathrm{~N}$ & $0.96 \mathrm{~N}$ & $0.55 \mathrm{~N}$ & $0.8 \mathrm{~N}$ \\
\hline 47.75 & $1.0 \mathrm{~N}$ & $1.19 \mathrm{~N}$ & $0.68 \mathrm{~N}$ & $1.0 \mathrm{~N}$ \\
\hline 57.30 & $1.2 \mathrm{~N}$ & $1.43 \mathrm{~N}$ & $0.82 \mathrm{~N}$ & $1.2 \mathrm{~N}$ \\
\hline 66.85 & $1.4 \mathrm{~N}$ & $1.67 \mathrm{~N}$ & $0.95 \mathrm{~N}$ & $1.4 \mathrm{~N}$ \\
\hline 76.39 & $1.6 \mathrm{~N}$ & $1.91 \mathrm{~N}$ & $1.09 \mathrm{~N}$ & $1.6 \mathrm{~N}$ \\
\hline 85.94 & $1.8 \mathrm{~N}$ & $2.15 \mathrm{~N}$ & $1.23 \mathrm{~N}$ & $1.8 \mathrm{~N}$ \\
\hline 95.49 & $2.0 \mathrm{~N}$ & $2.39 \mathrm{~N}$ & $1.36 \mathrm{~N}$ & $2.0 \mathrm{~N}$ \\
\hline
\end{tabular}

(a)

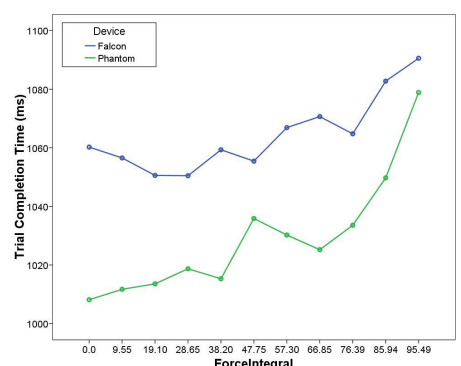

(b)

Fig. 4. (a) Force amplitudes calculated using the $F I$ value and the definite integral. (b) Force integral values by input device $(F I \times I D)$.

healthy subjects in this fundamental phase of the research in order to avoid the strong individual differences between different MS patients.

\subsection{Independent Variables and Design}

As we are primary comparing the haptic devices, the first independent variable is the input device $(I D)$. All the other remaining independent variables are those with regard to the selection experiment, similar as in [3]. The applied force bump is activated lateral to the movement direction.

The amplitude of the force bump over time $(T)$ follows a mathematical pattern such as a sine or a step function, which we define as the force shape $(S)$. For the shape $S=\sin _{[0, \pi]}, T=75 \mathrm{~ms}$ eleven amplitudes are considered. The amplitudes are converted to the integral of the force profile (see figure $3(\mathrm{~b})$ ) which we will refer to as the Force Integral $(F I)$. For the three other shapes the same FI is taken. An overview of the shapes and their amplitudes is given in figure 4(a).

A mixed design was applied: a repeated measures within-participant design for all independent variables was used except for input device $I D$, which is a between-participant factor. We preferred a between-participant design over a within-participant as carry-over between these devices will be large for participants. The independent variables were: force integral $F I(0.0,9.55,19.10, \ldots)$; and shape $S\left(\sin _{[0, \pi]}, T=75 \mathrm{~ms}\right.$; sqr, $T=40 \mathrm{~ms}$; $\sin _{[0, \pi]}, T=110 \mathrm{~ms}$; and $\sin _{[0,2 \pi]}$, $T=75 \mathrm{~ms}$ ). A fully crossed design resulted in 44 combinations of $F I$ and $S$.

Each participant was randomly assigned to a device and performed the experiment in one session lasting about 25 minutes. This way nine participants were assigned to each device. The session consisted of five blocks with each block containing the 44 combinations ( $11 F I$ s and $4 S \mathrm{~s}$ ) repeated three times in a random order. For a total of 132 trials per block, this resulted in 660 trials per participant. Between each block, users were obliged to take a 15 seconds' break to minimise fatigue during the test. Before the experiment, participants were given all 44 conditions in random order to familiarise them with the task. 
For each selected target, the logged parameters (at 200Hz) include completion time, the actual position of the cursor, velocity, the exerted force bump and the number of clicks before a successful selection.

\subsection{Results}

Trial Completion Time First, we will investigate the learning effects. Repeated measures analysis of variance of the faultless trials, showed a main effect for Block $\left(\mathrm{F}_{4,64}=18.991, \mathrm{p}<.0001\right)$, post hoc comparisons showed that the learning effect continued throughout all the blocks: participants were still improving their performance, even in the last block. With regard to the input device $I D$ no interaction effect could be seen with Block $\left(\mathrm{F}_{4,64}=.155, \mathrm{p}=.960\right)$, from which we can conclude that the devices had a similar learning curve. As the learning effect did not influence the device and was present in all blocks, we will continue our repeated measures analysis of variance including all blocks.

Input device $I D\left(\mathrm{~F}_{1,16}=.256, \mathrm{p}=.620\right)$ did not show a main effect. Therefore we can conclude that we found no significant difference between the devices. However, we saw that the overall trial completion time for the Phantom was $1029 \mathrm{~ms}$ and the Falcon 1064ms. Even in if this result would become significant in a longitudinal study, such a small difference can be argued to be of less practical use. The interactions $S \times I D\left(\mathrm{~F}_{3,48}=.811, \mathrm{p}=.494\right)$ and $F I \times I D\left(\mathrm{~F}_{3,48}=\right.$ $1.07, \mathrm{p}=.385)$ were not significant and showed a very similar trend. The Falcon consequently performs slightly slower. Figure 4(b) illustrates how each device behaves according to the respective force integral values. Not only is the Falcon consequently slower but it appears that for the highest FI conditions the Falcon has a less strong trial completion time deterioration compared to the Phantom.

Similarly as in our previous work, shape $S\left(\mathrm{~F}_{3,48}=.952, \mathrm{p}=.423\right)$ did not show a significant main effect and force integral $F I\left(\mathrm{~F}_{10,160}=8.0, \mathrm{p}<.0001\right)$ did show a significant main effect. Post hoc comparisons for $F I$ showed that above a certain force integral value $(F I=85.94)$ the trial completion time deteriorated significantly $(\mathrm{p}<.01)$. This value, 85.94 , is somewhat higher than in our previous experiments [3].

Finally, with regard to error rates during the experiment (wrong clicks) no significant difference between the Phantom (3.3\%) and the Falcon (2.0\%) was found.

Velocity Analysis Although we found no statistical significant differences between the devices, we are interested in the velocity behaviour during a selection task. Figure 5 depicts typical velocity profiles respectively from the Phantom and the Falcon. The uppermost graph contains the evolution of the lateral velocity over time, the bottommost graph the longitudinal velocity in the highest amplitude condition $(F I=95.49)$.

With regard to the Phantom, from the uppermost graph in figure 5, we can learn that the force bump causes an oscillation lateral to the movement direction. For the bottommost graph we can see that the longitudinal velocity, 

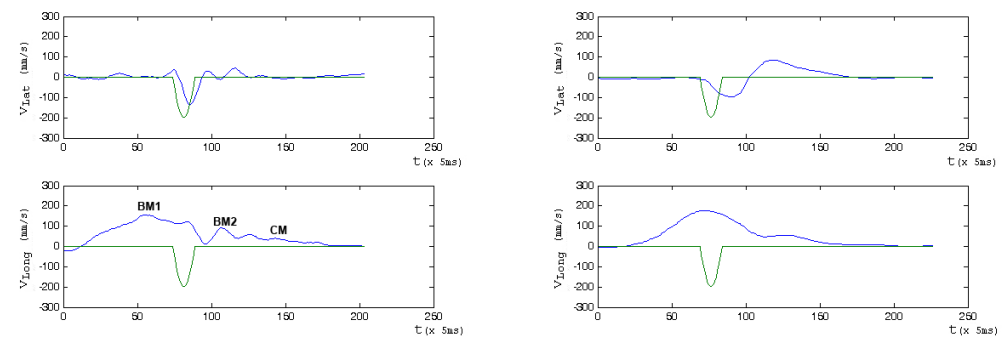

Fig. 5. Velocity profiles of the Phantom (left) and Falcon (right) during a selection trial $(F I=95.49)$. The top graph is the lateral velocity, the bottom graph represents the longitudinal.

does not completely behave according to the optimised initial impulse model of Meyer et al. [7]. The ballistic movement phase (BM1) is interrupted after the force bump, possibly to tackle the oscillation caused by the force feedback. A second but shorter ballistic movement (BM2) is initiated afterwards. Finally, the controlled movement $(\mathrm{CM})$ brings the cursor in an accurate way to the target. It can be assumed that the majority of the performance penalty is caused by the interruption of the ballistic movement. In our previous work [3] more graphs are available motivating this statement.

When considering the velocity behaviour of the Falcon, we see a similar effect, but the effect of the higher damping is clearly visible, as well. After the force bump, a more strongly damped oscillation occurs. Here again, the ballistic movement is interrupted, but in general in a less pronounced way. Keeping these figures in mind may help us to explain the behaviour in figure 4(b). At the no force condition $(F I=0)$ the Falcon appears to be (non-significantly) slower than the Phantom, which may not be surprisingly, because of the higher damping causing a lower longitudinal velocity (Average max. long. vel. for the Phantom: $\bar{v}_{\max }=60.12 \mathrm{~mm} / \mathrm{s}$; For the Falcon: $\left.\bar{v}_{\max }=42.03 \mathrm{~mm} / \mathrm{s}\right)$. Alternatively, the high forces cause less oscillation, and hence require a less severe interruption of the ballistic movement phase, which at its turn explains the lower performance deterioration in the higher force conditions $(F I=85.94$ and $F I=95.49)$.

\section{Subjective Comparison}

To further compare the difference between the haptic devices, we conducted a second experiment sounding out for the users' subjective experience with respect to the different devices. For this experiment, we used the same apparatus and applied the same experimental procedure as in the previous experiment. The force bump had a lateral force direction with $S=\sin _{[0, \pi]}, T=75 \mathrm{~ms}$ which was also present in the other experiment. The participants from the previous objective device experiment immediately participated in this experiment. Hence, a between-participant design was used. 


\subsection{Stimuli and Design}

For this experiment, we applied an adaptive staircase method [5]. In our case we will ask the users if they consider the current force as too strong. In the conducted experiment, we simultaneously interleaved two staircases: a 'one-up two-down' design starting from $2.0 \mathrm{~N}$ and a 'two-up one-down' design starting from $0.6 \mathrm{~N}$. This design ensures that the user approaches the threshold from two different sides. For the first four reversals the step size was set to $0.3 \mathrm{~N}$, thereafter a smaller step size for a resolution of $0.1 \mathrm{~N}$ has been taken for the next six reversals. Hence, the experiment ended after a total of ten reversals.

The user had to perform five selections after which we asked the question 'Do the forces complicate the execution of the selection task in any way?' The user had to answer 'yes' or 'no' using the keyboard with the non-dominant hand and depending on the answer and staircase, the force strength was changed accordingly. Each participant performed the experiment in one session lasting about 15 minutes depending on the time necessary to complete the 10 reversals. We logged the current strength value, step size and amount of reversals so far.

\subsection{Results}

For each device, for each participant, the mean force strength value for both staircases was calculated by averaging the force strengths of the last 6 resolution reversals. Averaging these values for the Phantom, we found that the one-up twodown staircase (started at $2.0 \mathrm{~N})$ had an average of $1.45 \mathrm{~N}(\mathrm{sd}=0.48)$ and the two-up one down staircase (coming from $0.6 \mathrm{~N})$ an average of $1.05 \mathrm{~N}(\mathrm{sd}=0.35)$, which results in an overall average of $1.25 \mathrm{~N}(\mathrm{sd}=0.41)(F I=59.68)$. For the Falcon we found that the one-up two-down staircase (started at $2.0 \mathrm{~N}$ ) had an average of $1.64 \mathrm{~N}$ ( $\mathrm{sd}=0.71)$ and the two-up one down staircase (coming from $0.6 \mathrm{~N})$ an average of $1.17 \mathrm{~N}(\mathrm{sd}=0.60)$, which results in an overall average of $1.41 \mathrm{~N}(\mathrm{sd}=0.66)(F I=67.32)$.

The Falcon thus resulted in a higher value for the mean force strength than the Phantom, respectively $1.41 \mathrm{~N}$ and $1.25 \mathrm{~N}$. The higher damping for the Falcon might influence the perception and hence cause this result. Moreover, Falcon participants had to answer the question $27 \%$ more often. This could imply that forces are less distinguishable with the Falcon. In order to justify this finding, a force discrimination experiment comparing the devices would be necessary.

\section{Discussion and Conclusion}

In order to investigate in what respect the Falcon haptic device may serve as a low-cost alternative for the Phantom, we compared these haptic devices. We discussed the differences between the devices and deduced their most important mechanical characteristics. This calculation learned us that the Falcon has a higher damping than the Phantom. The devices were then compared in a target acquisition task using an objective and subjective experiment. In the objective experiment we found no significant difference for the trial completion time 
between the devices. Note that this is no other conclusion than in previously conducted haptic device comparison experiments, comparing the Phantom to other haptic devices $[9,6]$. We did notice that the velocity profile due to the force feedback behaves differently because of the higher damping of the Falcon. The subjective experiment showed that the Falcon allows for higher force amplitudes than the Phantom, probably due to higher damping which decreases the 'correct' discrimination of the applied forces.

We can conclude that the higher damping of the Falcon with regard to the Phantom plays a role in the perception and behaviour of the user during a target acquisition task, but in practice, the differences in performance are small and

non-significant. This may strengthen our idea of further investigating the use of the Falcon in the context of our rehabilitation project. In a next step, we can apply and compare the Falcon in a practical setup with patients, and investigate for what exercises it is most suitable or not. This may possibly reveal other issues as well, such as the limited workspace, shifting of the device, or differences between the grip (knob or stylus) of the devices. A force discrimination experiment between the two devices (including also patients) may be useful, as well.

\section{Acknowledgments}

Part of the research at EDM is funded by the ERDF and the Flemish government. This research was funded through the INTERREG-IV program (project 4-BMG-II-1-84 and IVA-VLANED-1.14, Euregio Benelux).

\section{References}

1. Chortis, A., Standen, P.J., Walker, M.: Virtual reality system for upper extremity rehabilitation of chronic stroke patients living in the community. In: International Conference Series on Disability, Virtual Reality and Associated Technologies (2008)

2. De Boeck, J., Alders, G., Gijbels, D., De Weyer, T., Raymaekers, C., Coninx, K., Feys, P.: The learning effect of force feedback enabled robotic rehabilitation of the upper limbs in persons with MS - a pilot study. In: ENACTIVE'08. pp. $117-122$

3. De Boeck, J., Vanacken, L., Coninx, K.: Target aquisition with force feedback: The effect of different forces on the user's performance. In: HAID'09. pp. 11-20

4. Deutsch, J.E., Latonio, J., Burdea, G.C., Boian, R.: Post-stroke rehabilitation with the rutgers ankle system: A case study. Presence 10(4), 416-430 (2001)

5. Leek, M.: Adaptive procedures in psychophysical research. Perception \& Psychophysics 63(8), 1279-1292 (2001)

6. M. Harders, A. Barlit, K.A.M.S.G.S.: Comparing 6DOF Haptic Interfaces for Application in 3D Assembly Task. In: Proc. of Eurohaptics 2006. pp. 523-526 (2006)

7. Meyer, D., Abrams, R., Kornblum, S., Wright, C., Smith, J.: Optimality in human motor performance: Ideal control of rapid aiming movements pp. 340-370 (1988)

8. Pintelon, R., Guillaume, P., Rolain, Y., Schoukens, J., H, V.: Parametric idenitifcation of transfer-functions in the frequency-domain - a survey. In: IEEE Transactions on Automatic Control. vol. 39, pp. 2245-2260 (1994)

9. Yu, W., Brewster, S.: Comparing two haptic interfaces for multimodal graph rendering. In: HAPTICS '02. pp. 3-9 (2002) 